\title{
Métodos para avaliação in vitro da atividade antimicrobiana de plantas medicinais: a necessidade da padronização
}

\author{
Methods for in vitro evaluating antimicrobial activity of medicinal \\ plants: the need for standardization
}

Recebido em: $11 / 08 / 2017$

Aceito em: $20 / 11 / 2017$
Tatiane Roquete AMPARO; Vanessa Cristina Carvalho BRAGA; Janaína Brandão SEIBERT; Gustavo Henrique Bianco de SOUZA;

Luiz Fernando Medeiros TEIXEIRA

Universidade Federal de Ouro Preto, Escola de Farmácia S/N, Morro do Cruzeiro, CEP 35400-000. Ouro Preto, MG, Brasil.E-mail: tatianeroquete@yahoo.com.br

\section{ABSTRACT}

The increasing incidence of resistant pathogens to current drugs encourages the search for new antimicrobial agents. In this context, medicinal plants are an important source of new drugs. There are several methods for evaluating the antibacterial and antifungal activity of extracts, fractions, essential oils and isolated substances from the plant. The most known include diffusion, dilution and bioautography methods. The purpose of this paper is to present the most used methods currently, along with their advantages, disadvantages and interfering factors. Among the works available in the SciELO database, covering the last ten years, only $4,4 \%$ of research on medicinal plants are related to the antimicrobial activity. The most used method is microdilution $(57,9 \%)$, the best recommended due to high sensitivity, the minimum quantity of reagents and sample and the possibility of a more significant number of replicates. In articles that used this method, differences were observed in factors that may affect the results. Thereby, there is the need for standardization of methods used by researchers to facilitate obtaining comparable and reproducible results. To achieve comparable and reproducible results, there is the need to standardize the methods used by the researchers. It is recommended to use as reference the standards established by CLSI for culture medium and inoculum concentration in the tests. It also recommended the inclusion of negative control of the solubilization of the samples with quantification of microbial growth to avoid interference with the results.

Keywords: medicinal plants; microbiology; microbial sensitivity tests

\section{RESUMO}

A crescente incidência de patógenos resistentes aos medicamentos atuais incentiva a busca de novos agentes antimicrobianos. Neste contexto, as plantas medicinais se destacam, sendo uma importante fonte de novos fármacos. Existem diversos métodos para avaliar a atividade antibacteriana e antifúngica de extratos, frações, óleos essenciais e substâncias isoladas de vegetais. Os mais conhecidos incluem métodos de difusão, diluição e bioautografia. A proposta desse trabalho é apresentar os métodos mais utilizados atualmente, juntamente com suas vantagens, desvantagens $\mathrm{e}$ fatores interferentes. Entre os artigos indexados na biblioteca SciELO, abrangendo os últimos dez anos, somente 4,4\% das pesquisas com plantas medicinais estão relacionadas com atividade antimicrobiana. O método mais utilizado foi a microdiluição $(57,9 \%)$, o mais recomendado devido à alta sensibilidade, à quantidade mínima de reagentes e amostra e à possibilidade de um maior número de réplicas. Nos trabalhos que utilizaram esse método, foram verificadas divergências de fatores que podem interferir nos resultados. A fim de facilitar a obtenção de resultados comparáveis e reprodutíveis, se faz necessária a padronização dos métodos utilizados pelos pesquisadores. Recomenda-se utilizar como 
referência as normas estabelecidas pelo CLSI para meio de cultura e concentração de inóculo nos testes. Além disso, também se recomenda a inclusão de um controle negativo da forma de solubilização das amostras, com quantificação do crescimento microbiano, para evitar a interferência nos resultados.

Palavras-chaves: plantas medicinais; microbiologia; testes de sensibilidade microbiana

\section{INTRODUÇÃO}

As plantas são utilizadas na medicina tradicional desde as antigas civilizações e essa importância terapêutica incentiva as pesquisas para desenvolvimento de novos fármacos (1). $\mathrm{O}$ interesse dos pesquisadores na avaliação da atividade antimicrobiana de plantas medicinais tem sido despertado pela aceitação dessas espécies como alternativa no tratamento de doenças e pelo aumento de microrganismos resistentes aos antibióticos disponíveis (2).

Além disso, também deve ser ressaltada a importância dos estudos in vitro sobre atividade antimicrobiana em um país de grande biodiversidade como o Brasil, pois podem ser utilizados para a primeira triagem na descoberta da atividade farmacológica das espécies (3).

A eficácia antimicrobiana de extratos vegetais, óleos essenciais e compostos isolados, como alcaloides, flavonoides, lactonas e terpenos, tem sido relatada (4). Diversos métodos para teste de atividade antimicrobiana de produtos naturais estão disponíveis na literatura, incluindo avaliações qualitativas (triagens) e/ou quantitativas, com determinação da menor concentração capaz de inibir o crescimento microbiano (concentração inibitória mínima-CIM) (5).

Os métodos de avaliação de susceptibilidade de antibióticos utilizados na clínica são padronizados por normas estabelecidas por órgãos como o CLSI (Clinical and Laboratory Standards Institute), o mais adotado internacionalmente. Além desse, também existem outros como: British Society for Antimicrobial Chemotherapy (BSAC) e European Committee on Antimicrobial Susceptibility Testing (EUCAST) e atualmente Brazilian Commitee on Antimicrobial Susceptibility Testing (BrCAST).

Os produtos naturais de constituição complexa, possuem características físico-químicas diferentes dos antibióticos comerciais e dessa forma, são necessárias modificações nas normas propostas e não existe uma padronização dos métodos de avaliação da atividade antimicrobiana de extratos vegetais e óleos essenciais.

O objetivo deste trabalho é apresentar os principais métodos utilizados nos últimos dez anos, principalmente na pesquisa brasileira, bem como os principais fatores interferentes no método mais utilizado. Dessa maneira, contribuir como fonte de pesquisa para o desenvolvimento de futuros trabalhos e chamar a atenção para a necessidade de uma padronização dos métodos.

\section{MÉTODOS}

A pesquisa foi realizada no banco de dados Scientific Electronic Library Online - SciELO com as palavras-chaves Medicinal plants e Antimicrobial activity of medicinal plants. Foram incluídos somente artigos originais abordando atividade antibacteriana e/ou antifúngica in vitro, publicados no período de 2007 a 2016. A busca de dados foi realizada no dia 06 de junho de 2016. A discussão dos resultados encontrados foi realizada com base em artigos da área, sem restrição de biblioteca científica nem período de tempo.

\section{RESULTADOS E DISCUSSÃO}

Pesquisas realizadas com plantas medicinais. A resistência microbiana, causa de preocupação global, dificulta o tratamento de doenças infecciosas, prolongando o tempo da doença e aumentando a mortalidade e os custos. Atualmente tem se relatado o aumento de microrganismos resistentes aos fármacos atuais juntamente com uma escassez na descoberta de novos antimicrobianos (6).

Apesar da necessidade de novos medicamentos, existem ainda poucas pesquisas a partir de plantas medicinais, que são uma importante fonte de novos fármacos. No período de 2007 a 2016, foram indexados, na Scielo, 853 artigos sobre o referido assunto, sendo que somente $38(4,4 \%)$ abordavam atividade antimicrobiana. Com o objetivo de apresentar e discutir os métodos mais utilizados atualmente, esses 38 artigos foram compilados (Tabela 1).

Métodos de avaliação da atividade antimicrobiana. Existem diversos métodos de avaliação da atividade antimicrobiana de produtos naturais. Devido às varia- 
ções, como técnica aplicada, microrganismos e cepas utilizadas, concentração do inóculo, meio de cultura e características dos extratos, não existe padronização de um método para a avaliação (3). Os métodos de avaliação de atividade antimicrobiana in vitro utilizados nos artigos analisados foram bioautografia, difusão e diluição (Tabela 1).

A bioautografia é utilizada para indicar os constituintes bioativos de extratos vegetais. Esse método é acoplado a técnicas cromatográficas (cromatografia em camada delgada - CCD) e pode ser realizado de forma direta, indireta ou por imersão (7). A bioautografia direta envolve aplicação da suspensão microbiana diretamente sobre a superfície da placa cromatográfica; na forma indireta, o cromatograma desenvolvido é colocado sobre a superfície de um ágar pré-inoculado por um período específico para permitir a difusão (7). $\mathrm{Na}$ análise por imersão, a placa cromatográfica é imersa pelo ágar para difusão das substâncias para o meio de cultura (7).

Tabela 1: Artigos sobre atividade antimicrobiana de plantas medicinais indexados na SciEL0 no período de 2007 a 2016

\begin{tabular}{|c|c|c|c|c|c|c|c|c|}
\hline \multirow{4}{*}{ Referência } & \multirow{4}{*}{ Amostra } & \multirow{4}{*}{ Atividade } & \multicolumn{6}{|c|}{ Método utilizado } \\
\hline & & & \multirow{3}{*}{ Bioautografia } & \multicolumn{2}{|c|}{ Difusão } & \multicolumn{3}{|c|}{ Diluição } \\
\hline & & & & \multirow{2}{*}{ Discos } & \multirow{2}{*}{ Poços } & \multirow{2}{*}{ Ágar } & \multicolumn{2}{|c|}{ Caldo } \\
\hline & & & & & & & Macro & Micro \\
\hline (8) & Extrato & Antibacteriana & & & & $X(T)$ & & $X($ CIM $)$ \\
\hline (9) & Óleo essencial & Antibacteriana & & & & & & $X($ CIM $)$ \\
\hline (10) & Extratos e óleos essenciais & Antibacteriana & & & & & & $X($ CIM) \\
\hline (11) & Extratos & Antibacteriana & & & $X(T)$ & & & $X($ CIM $)$ \\
\hline (12) & Extratos & Antibacteriana & & & & & & X (CIM) \\
\hline (13) & Extratos & Antimicrobiana* & & & & & & $\mathrm{X}(\mathrm{CIM})$ \\
\hline (14) & Óleo essencial & Antibacteriana & & $\mathrm{X}(\mathrm{T})$ & & & & X (CIM) \\
\hline (15) & Extratos & Antibacteriana & & & & & & $X($ CIM $)$ \\
\hline$(16)$ & Extratos & Antibacteriana & & & & & & $X($ CIM) \\
\hline (17) & Óleos essenciais & Antibacteriana & & $\mathrm{X}(\mathrm{T})$ & & & & \\
\hline (18) & Extrato & Antibacteriana & & $x(T)$ & & $X($ CIM $)$ & & \\
\hline (19) & Óleo essencial & Antibacteriana & & & $X(T)$ & & & \\
\hline$(20)$ & Extrato & Antibacteriana & & $X(T)$ & & & & $X($ CIM $)$ \\
\hline (21) & Extratos & Antimicrobiana* & & & $\mathrm{X}(\mathrm{T})$ & & & \\
\hline (22) & Frações & Antibacteriana & $x$ & & $X(T)$ & & & \\
\hline$(23)$ & Extrato & Antibacteriana & & $X(T)$ & & & & $X($ CIM $)$ \\
\hline$(24)$ & Óleo essencial & Antimicrobiana* & & & & & & $X($ CIM $)$ \\
\hline$(25)$ & Extratos & Antibacteriana & & & & & & $X($ CIM $)$ \\
\hline (26) & Extratos e frações & Antimicrobiana* & & & & & & $X($ CIM $)$ \\
\hline (27) & óleos essenciais (metabólitos) & Antibacteriana & & & & & $X(C I M)$ & \\
\hline$(28)$ & Óleos essenciais & Antibacteriana & & $X(T)$ & & & & \\
\hline (29) & Metabólitos sintetizados & Antimicrobiana* & & & & & & $X(C I M)$ \\
\hline$(30)$ & Extratos e óleos essenciais & Antifúngica & & & & & & $X($ CIM $)$ \\
\hline$(31)$ & Extrato e fração & Antibacteriana & & & $X($ CIM) & & & \\
\hline (32) & Extratos & Antimicrobiana* & & & & $X($ CIM $)$ & & \\
\hline (33) & Extratos e óleos essenciais & Antifúngica & & & & & & $\mathrm{X}(\mathrm{CIM})$ \\
\hline (34) & Extratos & Antifúngica & & & & & & $X(C I M)$ \\
\hline (35) & Extratos & Antimicrobiana* & & & & $X($ CIM $)$ & & \\
\hline$(36)$ & Óleo-resina & Antibacteriana & & $X(C I M)$ & & & & \\
\hline (37) & Extratos & Antimicrobiana* & & $X($ CIM $)$ & & & & \\
\hline
\end{tabular}




\begin{tabular}{|c|c|c|c|c|c|c|c|c|}
\hline \multirow{4}{*}{ Referência } & \multirow{4}{*}{ Amostra } & \multirow{4}{*}{ Atividade } & \multicolumn{6}{|c|}{ Método utilizado } \\
\hline & & & \multirow{3}{*}{ Bioautografia } & \multicolumn{2}{|c|}{ Difusão } & \multicolumn{3}{|c|}{ Diluição } \\
\hline & & & & \multirow{2}{*}{ Discos } & \multirow{2}{*}{ Poços } & \multirow{2}{*}{ Ágar } & \multicolumn{2}{|c|}{ Caldo } \\
\hline & & & & & & & Macro & Micro \\
\hline (41) & Extratos e apolares & Antimicrobiana* & & $X(T)$ & & $X($ CIM $)$ & & \\
\hline (42) & óleos essenciais & Antimicrobiana* & & $X($ CIM) & & & & \\
\hline (43) & Extratos & Antibacteriana & & & & $X($ CIM $)$ & & \\
\hline (44) & Extrato & Antibacteriana & & & X (CIM) & & & \\
\hline (45) & Extratos e isolados & Antibacteriana & $x$ & & $X(T)$ & $X(C I M)$ & & X (CIM) \\
\hline
\end{tabular}

*Atividade antifúngica e antibacteriana; T: Triagem; CIM: Determinação da concentração inibitória mínima

A bioautografia possui como principais vantagens a pequena quantidade de amostra e o direcionamento para identificação e isolamentos de constituintes bioativos (7). Apesar disso, sua baixa utilização (5,2\% dos artigos analisados) (Tabela 1) pode ser devida a limitações como necessidade de conhecimentos em cromatografia, baixa sensibilidade e reprodutibilidade.

No método de difusão em ágar, a amostra a ser testada é disposta em discos de papel ou orifícios (poços) perfurados no meio sólido, previamente inoculado com o microrganismo. Os resultados são expressos como média do diâmetro do halo de inibição de crescimento do microrganismo, gerado pela difusão da amostra após incubação (3).

Tabela 2: Características presentes no método de microdiluição utilizado nos artigos indexados na SciEL0 no período de 2007 a 2016.

\begin{tabular}{|c|c|c|c|}
\hline Referência & Meio de cultura & Solubilização * * & $\begin{array}{l}\text { Concentração do } \\
\text { inóculo (UFC/mL) }\end{array}$ \\
\hline (8) & Mueller-Hinton & DMSO $0,2 \%$ & $5 \times 10^{7}$ \\
\hline (9) & Mueller-Hinton & Sem solubilização & $1 \times 10^{5}$ \\
\hline (10) & Middlebrook $7 \mathrm{H} 9$ & DMSO $2,5 \%$ & $6 \times 10^{6}$ \\
\hline (11) & Mueller-Hinton & DMSO 1\% & $5 \times 10^{5}$ \\
\hline (12) & Mueller-Hinton & Etanol $25 \%$ & $1 \times 10^{4}$ \\
\hline (13) & * & * & * \\
\hline (14) & BHI & Tween $802 \%$ & * \\
\hline (15) & Caldo nutriente & DMSO 2,5\% & $5 \times 10^{5}$ \\
\hline (16) & Mueller-Hinton & DMSO $2,0 \%$ & $1,25 \times 10^{6}$ \\
\hline (20) & Mueller-Hinton & * & $5 \times 10^{7}$ \\
\hline (23) & caldo C. Rivas & Sem solubilização & $6,7 \times 10^{5}$ \\
\hline (24) & BHI e Saboraud & Tween $* \%$ & $1 \times 10^{5}$ \\
\hline (25) & meio LB & Sem solubilização & * \\
\hline (26) & Mueller-Hinton & * & * \\
\hline (29) & Triptona Soja & * & $1 \times 10^{8}$ \\
\hline$(30)$ & * & * & $1 \times 10^{5}$ \\
\hline (33) & * & Tween $0,001 \%$ & $5 \times 10^{4}$ \\
\hline (34) & RPMI 1640 & Diclorometano $25 \%$ & $2,5 \times 10^{3}$ \\
\hline (38) & Mueller-Hinton & DMSO *\% & $1 \times 10^{4}$ \\
\hline (39) & * & * & * \\
\hline$(40)$ & * & DMSO *\% & * \\
\hline$(45)$ & * & * & * \\
\hline
\end{tabular}

*Não especificado no texto, ${ }^{* *}$ Concentração final (\%) do solvente ou tensoativo utilizado para solubilização das amostras no teste

$\mathrm{O}$ método de difusão em ágar foi utilizado em $44,7 \%$ do total dos artigos analisados (Tabela 1). A ampla utilização desse método é devida a sua simplicidade e baixo custo, porém, esse método não é indicado para determinação da CIM. Esse fato é devido a fatores que interferem na difusão dos compostos no meio de cultura, como espessura do ágar, volume aplicado e propriedades físico-químicas das amostras analisadas $(46,47)$. Dessa 
forma, permite apenas uma triagem da atividade de produtos naturais, direcionando as análises para determinação da CIM, como foi utilizada em 38,9\% dos artigos que aplicaram esse método.

Entre as variáveis desse método, a difusão com dis$\cos$ foi a mais utilizada $(58,8 \%)$ que a difusão a partir de poços. A difusão com discos é utilizada principalmente para óleos vegetais, porém, o método em poços é considerado mais sensível para extratos. Neste caso, a diferença entre os métodos é explicada pela adesão de algumas substâncias ao disco de papel que impede a difusão para o ágar. A adesão é devido à propriedade hidrofílica da superfície do disco que possui grupos hidroxila livres presentes em cada um dos resíduos de glicose (5).

Os métodos de diluição foram os mais utilizados nos artigos analisados e, dentre esses, somente 23,3\% utilizaram a diluição em ágar e a maioria optou pela diluição em caldo $(76,7 \%)$.

$\mathrm{Na}$ diluição em ágar, os extratos são diluídos no meio de cultura fundido em várias concentrações e os microrganismos são inoculados após a gelificação do meio. Esse método de diluição é considerado mais sen- sível que a difusão em ágar, porém é menos sensível que a diluição em caldo (47).

A diluição em caldo é considerada o método mais sensível para determinação da CIM e pode ser feita em tubos (macrodiluição) ou em placas de 96 poços (microdiluição). A microdiluição foi o método mais utilizado $(57,9 \%)$. Além da sensibilidade, esse método possui vantagens como reprodutibilidade, pequena quantidade de amostra requerida e possibilidade de teste simultâneo de um grande número de amostras.

A comparação entre métodos distintos já foi realizada por vários autores. Alves e cols (2008) e Scorzoni e cols. (2007) compararam o método de microdiluição com métodos de difusão em ágar e concluíram que o primeiro é mais recomendado para determinação da CIM, pois é mais sensível e reprodutível $(46,48)$.

A microdiluição também foi considerada o melhor método para avaliação da atividade antimicrobiana de plantas de uso medicinal (47). Os autores compararam essa técnica com a difusão em ágar e a macrodiluição em caldo e, além disso, também analisaram os resultados da microdiluição por diferentes reveladores.

\section{A}

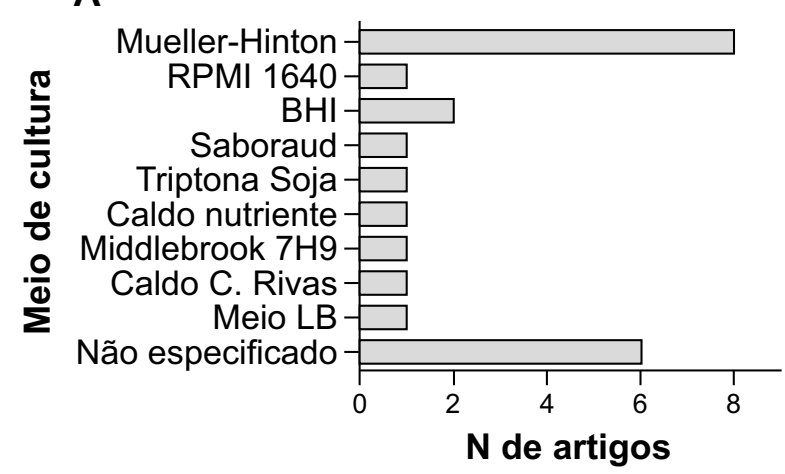

B

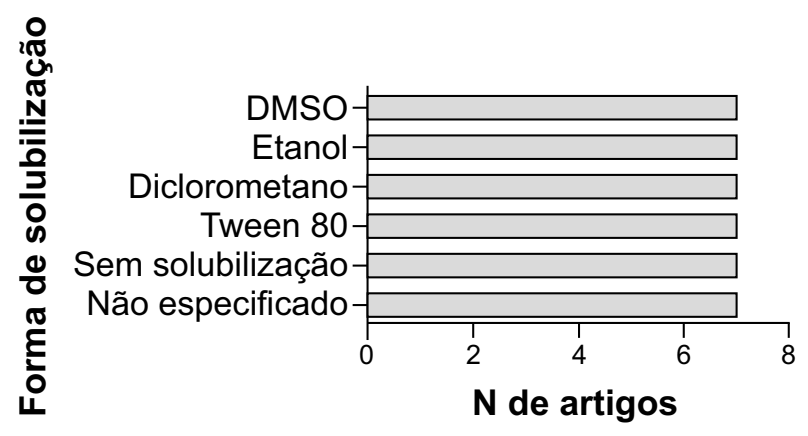

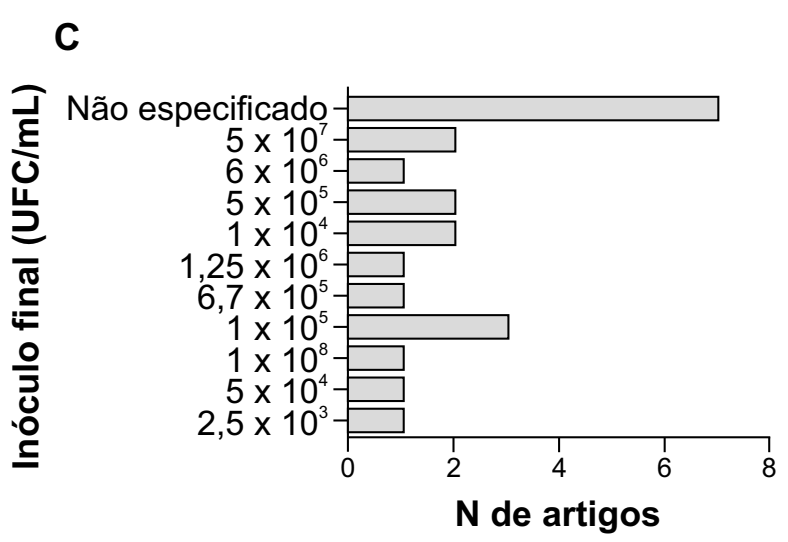

Figura 1. Variações dos fatores interferentes no método de microdiluição utilizado nos artigos indexados na SciEL0 no período de 2007 a 2016 : meio de cultura (A), forma de solubilização das amostras (B) e concentração final do inóculo (C). 
No entanto, não foi encontrado, na Scielo, qualquer estudo considerando a interferência de outros fatores variáveis da microdiluição. Dessa forma, diante da importância e da ampla utilização, foi realizada a análise das variáveis encontradas nos artigos que utilizaram esse método.

\section{Análise comparativa do método de microdilui-} ção. Os estudos que utilizaram a microdiluição, apesar de tratarem da mesma metodologia, utilizaram características diferentes (Tabela 2). Visando facilitar a observação da divergência dos fatores que podem interferir nos resultados do teste, sua prevalência foi sumarizada na Figura 1.

Um dos fatores interferentes é o meio de cultura utilizado, que deve favorecer o crescimento dos microrganismos e não conter substâncias antagônicas ao antimicrobiano estudado (3). A escolha do meio deve considerar o tipo de microrganismo e os indicados pelo CLSI são: caldo Mueller-Hinton, para bactérias e RPMI 1640 para leveduras $(49,50)$.

A maioria dos artigos analisados abordou atividade antibacteriana, e o caldo Mueller-Hinton foi o meio de cultura mais utilizado (36,4\%, Figura 1-A). O caldo Mueller-Hinton permite crescimento satisfatório da maioria dos patógenos e, além disso, pode ser suplementado, com por exemplo sangue animal, sais e açúcares, para promover o crescimento de bactérias fastidiosas (49).

Outro fator é a forma de solubilização dos extratos ou frações para adição no meio de cultura. O solvente pode inibir o crescimento microbiano e levar a resulta- dos falso-positivos. A dificuldade de solubilização é uma das características dos produtos naturais que impede que a metodologia proposta pelo CLSI seja seguida integralmente, exigindo modificações (51).

Nos artigos analisados, o DMSO (dimetilsulfóxido) foi o solvente mais utilizado para solubilização das amostras (31,8\%) (Figura 1-B). Esse solvente permite uma boa solubilização de diversas substâncias, mas pode inibir o crescimento microbiano.

Ansel e cols. (1969) relataram o efeito inibitório de diferentes concentrações de DMSO sobre o crescimento de Escherichia coli, Pseudomonas aeruginosa e Bacillus megaterium. Mesmo as menores concentrações (1 e $2 \%$ ) inibiram de 7,5 a $18,7 \%$ do crescimento das bactérias (52).

Dessa forma, independente do solvente utilizado, é importante realizar um controle negativo com quantificação do crescimento na concentração de solvente empregada. Isso porque a inibição, até em menores intensidades, pode interferir nos resultados. A maioria dos trabalhos encontrados na literatura descrevem a realização do controle negativo, mas não quantificam o crescimento. Além disso, o controle deve ser realizado em todos os testes, pois a inibição pode variar com as cepas utilizadas.

Os cuidados e dificuldades relacionados à solubilização das amostras não são encontrados em casos de óleos essenciais, formulações e extratos aquosos que podem ser adicionados diretamente ao meio de cultura líquido.

Tabela 3. Resultados de atividade antimicrobiana de $\alpha$ - e $\beta$-amirina e variáveis dos estudos encontrados na literatura

\begin{tabular}{|c|c|c|c|c|}
\hline Referência & $\begin{array}{l}\text { Meio de cultura/ } \\
\text { solvente* }\end{array}$ & $\begin{array}{l}\text { Concentração final } \\
\text { do inóculo } \\
\text { (UFC/mL) }\end{array}$ & $\begin{array}{l}\text { Substância } \\
\text { testada }\end{array}$ & $\begin{array}{l}\text { Microrganismos (CIM } \mu \mathrm{g} / \\
\mathrm{mL})\end{array}$ \\
\hline (53) & $\begin{array}{l}\text { Mueller-Hinton/ DMSO } \\
5 \%\end{array}$ & $5 \times 10^{5}$ & $\alpha$-amirina & $\begin{array}{l}\text { S. aureus }(>100) \\
\text { E. faecalis }(>100) \\
\text { E. coli }(>100)\end{array}$ \\
\hline (54) & $\begin{array}{l}\text { Mueller-Hinton/ } \mathrm{MeOH} \\
50 \%\end{array}$ & $5 \times 10^{5}$ & $\alpha$-amirina & S. aureus (64) \\
\hline (55) & Mueller-Hinton & $5 \times 10^{5}$ & $\beta$-amirina & $\begin{array}{l}\text { S. aureus }(>200) \\
\text { S. enteritidis }(>200) \\
\text { E. coli }(>200) \\
\text { E. faecalis }(>200)\end{array}$ \\
\hline$(56)$ & BHI/ DMSO 2,5\% & $2,5 \times 10^{6}$ & $\beta$-amirina & $\begin{array}{l}\text { S. aureus (256) } \\
\text { E. coli }(1024) \\
\text { K. pneumoniae (1024) } \\
\text { P. aeruginosa }(1024)\end{array}$ \\
\hline
\end{tabular}

*Concentração final (\%) do solvente ou tensoativo utilizado para solubilização das amostras no teste 
A concentração do inóculo também pode interferir no resultado e foi possível observar uma grande variação nos estudos analisados (Figura 1-C). A atividade antimicrobiana é dependente da concentração final de microrganismos no teste (inóculo), portanto é necessário sua padronização e a quantidade inoculada deverá ser estabelecida para cada método desenvolvido (3). O CLSI padroniza como concentração final no teste aproximadamente $5 \times 10^{5} \mathrm{UFC} / \mathrm{mL}$ para bactérias e para leveduras 0,5 a $2,5 \times 10^{3} \mathrm{UFC} / \mathrm{mL}$. Somente $18,2 \%$ dos artigos analisados utilizaram essas concentrações de inóculo padronizadas.

$\mathrm{Na}$ análise realizada também foi possível observar um grande número de artigos que não esclareceram no texto dados sobre os fatores interferentes. Uma descrição clara da metodologia empregada pode facilitar a reprodução dos resultados por outros pesquisadores.

A dificuldade de comparação entre os resultados de atividade antimicrobiana de plantas de uso medicinal é evidente. Diferentes grupos de trabalho podem obter resultados contraditórios devido à falta de uniformidade nas metodologias (48).

Um exemplo de divergência de resultados causada por fatores interferentes no método de diluição (concentração de inóculo, forma de solubilização das amostras, meio de cultura e cepas utilizadas) é demonstrado para a atividade antimicrobiana dos triterpenos $\alpha$ - e $\beta$-amirina (Tabela 3).

\section{CONCLUSÃO}

A correta seleção dos bioensaios é um importante passo no estudo da atividade biológica de produtos naturais e esses testes devem ser simples, sensíveis e reprodutíveis (57). Para avaliação da atividade antimi- crobiana, os métodos mais utilizados e recomendados são difusão em ágar, para triagem da atividade, e microdiluição, para determinação da CIM.

O método da microdiluição é considerado sensível e reprodutível, porém foram verificadas divergências de fatores, como meio de cultura, concentração de inóculo e solubilização das amostras, que podem interferir nos resultados. Essa ausência de padronização da metodologia dificulta a comparação e reprodução dos resultados.

A solubilidade é a principal característica dos extratos vegetais que dificulta a total aplicação das normas do CLSI, padrão mais aceito internacionalmente (51). Os extratos vegetais são amostras complexas de propriedades físico-químicas particulares e é difícil padronizar sua solubilização para os testes de atividade antimicrobiana. Sendo assim, são utilizados diferentes solventes e tensoativos que podem inibir o crescimento microbiano.

O presente trabalho representa uma fonte de referências para escolha das metodologias de avaliação da atividade antimicrobiana de plantas medicinais. Além disso, os resultados e discussões apresentados destacam a necessidade da padronização dos métodos utilizados pelos pesquisadores. Recomenda-se a utilização das normas estabelecidas pelo CLSI para meio de cultura e concentração de inóculo nos testes. Além disso, também se recomenda a inclusão de um controle negativo da forma de solubilização das amostras, com quantificação do crescimento microbiano, para evitar a interferência nos resultados.

\section{AGRADECIMENTOS}

À Universidade Federal de Ouro Preto, à CAPES e à FAPEMIG.

\section{REFERÊNCIAS}

1. Pires IFB, Souza AA, Feitosa MHA, Costa SM. Plantas medicinais como opção terapêutica em comunidade de Montes Claros, Minas Gerais, Brasil. Rev Bras Plantas Med. 2014;16(2):426-433. DOI: 10.1590/1983084X/12_089

2. Das K, Tiwari RKSE, Shrivastava DK. Techniques for evaluation of medicinal plant products as antimicrobial agent: Current methods and future trends. J Med Plants Res. 2010; 4(2): 104-111. DOI: 10.5897/JMPR09.030

3. Ostrosky EA, Mizumoto MK, Lima MEL, Kaneko TM, Nishikawa SO, Freitas BR. Métodos para avaliação da atividade antimicrobiana e determinação da Concentração Mínima Inibitória (CMI) de plantas medicinais. Rev Bras Farmacogn. 2008;18(2):301-307. DOI: 10.1590/ S0102-695X2008000200026 
4. Ríos JL, Recio MC. Medicinal plants and antimicrobial activity. J Ethnopharmacol. 2005;100(1-2):80-84. DOI: 10.1016/j.jep.2005.04.025

5. Valgas C, Souza SM, Smânia EFA, Smânia Jr A. Screening methods to determine antibacterial activity of natural products. Braz J Microbiol. 2007;38:369-380. DOI: 10.1590/S1517-83822007000200034

6. Jindal AK, Pandya K, Khan ID. Antimicrobial resistance: A public health challenge. MeD J Armed Forces India. 2015;71(2):178-181. DOI: 10.1016/j.mjafi.2014.04.011.

7. Kenny CR, Furey A, Lucey B. A post-antibiotic era looms: can plant natural product research fill the void? Br J Biomed Sci. 2015;72(4):191-200.

8. Barboza TJS, Ferreira AF, Ignacio ACPR, Albarello N. Antimicrobial activity of Anonna mucosa (Jacq.) grown in vivo and obtained by in vitro culture. Braz J Microbiol. 2015;46(3):785-789. DOI: 10.1590/S1517838246320140468

9. Sagave L, Gressler LT, Flores FC, Silva CB, Vargas APC, Lovato M, Sangioni LA, Pötter L, Botton SA. Atividade de nanoformulações de Melaleuca alternifolia e terpinen-4-ol em isolados de Rhodococcus equi. Arq Bras Med Vet Zootec. 2015;67(1):221-226. DOI: 10.1590/16787454

10. Machado RRP, Dutra RC, Pittella F, Raposo NRB, Lesche B, Duarte RS, Soares GLG, Kaplan MAC. Screening antimycobacterial activity of Baccharis dracunculifolia, Centella asiatica, Lantana camara and Pterodon emarginatus. Rev Bras Plantas Med. 2015;17(4):891-899. DOI: 10.1590/1983-084X/14_148

11. Miranda JAL, Rocha JA, Araújo KM, Quelemes PV, Mayo SJ, Andrade IM. Atividade antibacteriana de extratos de folhas de Montrichardia linifera (Arruda) Schott (Araceae). Rev Bras Plantas Med. 2015;17(4):11421149. DOI: 10.1590/1983-084x/14_169

12. Fernandes AWC, Aquino SÁMC, Gouveia GV, Almeida JRGS, Costa MM. Atividade antimicrobiana in vitro de extratos de plantas do bioma caatinga em isolados de Escherichia coli de suínos. Rev Bras Plantas Med. 2015;17(4): 1097-1102. DOI: 10.1590/1983084x/14_159

13. Lima Neto GA, Kaffashi S, Luiz WT, Ferreira WR, Dias da Silva YSA, Pazin GV, Violante IMP. Quantificação de metabólitos secundários e avaliação da atividade antimicrobiana e antioxidante de algumas plantas selecionadas do Cerrado de Mato Grosso. Rev Bras Plantas Med. 2015;17(4):1069-1077. DOI: 10.1590/1983084X/14_161

14. Andrade VA, Almeida AC, Souza DS, Colen KGF, Macêdo AA, Martins ER, Fonseca FSA, Santos RL. Antimicrobial activity and acute and chronic toxicity of the essential oil of Lippia origanoides. Pesq Vet Bras. 2014;34(12):1153-1161. DOI: 10.1590/S0100736X2014001200002

15. Sharma R, Lall N. Antibacterial, antioxidant activities and cytotoxicity of plants against Propionibacterium acnes. South Afr J Sci. 2014;110(11-12):1-8.
16. Araújo SG, Alves LF, Pinto MEA, Oliveira GT, Siqueira EP, Ribeiro RIMA, Ferreira JMS, Lima LARS. Volatile compounds of Lamiaceae exhibit a synergistic antibacterial activity with streptomycin. Braz J Microbiol. 2014;45(4):1341-1347. DOI: $10.1590 / \mathrm{S} 1517-$ 83822014000400026

17. Rodriguez Flores C, Pennec A, Nugier-Chauvin C, Daniellou R, Herrera-Estrella L, Chauvin AL. Chemical Composition and Antibacterial Activity of Essential Oils Extracted from Plants Cultivated in Mexico. J Mex Chem Soc. 2014;58(4): 452-455.

18. Garcia APM, Orlanda JFF. Avaliação da atividade antimicrobiana in vitro do extrato bruto hidroalcoólico de Mangifera indica Linneau. Rev Cub Plantas Med. 2014;19(3): 189-198.

19. Ferreira SB, Dantas IC, Catao RMR. Avaliação da atividade antimicrobiana do óleo essencial de sucupira (Pterodon emarginatus Vogel). Rev Bras Plantas Med. 2014;16(2): 225-230. DOI: $10.1590 / \mathrm{S} 1516-$ 05722014000200009

20. Teles DG, Costa MM. Estudo da ação antimicrobiana conjunta de extratos aquosos de Tansagem (Plantago major 1., Plantaginaceae) e Romã (Punica granatum 1., Punicaceae) e interferência dos mesmos na ação da amoxicilina in vitro. Rev Bras Plantas Med. 2014;16(2):323328. DOI: 10.1590/1983-084X/11_123

21. Arrais LG, Lyra HFS, Batista DCA, Coutinho FN, Saraiva AM, Pereira RCA, Pisciottano MNC, Xavier HS, Melo SJ. Atividade antimicrobiana dos extratos metanólicos da raiz, caule e folhas de Croton pulegioides Baill. (Zabelê). Rev Bras Plantas Med. 2014;16(2):316-322. DOI: 10.1590/1983-084X/12_033

22. Aldana J, Tellez N, Gamboa F. Antimicrobial activity of fractions and subfractions of Elaeagia utilis against microorganisms of importance in dental caries. Acta Odontol Latinoam. 2013;26(2):104-111.

23. Vega-Menchaca MC, Verde-Star J, Oranday-Cárdenas A, Morales-Rubio ME, Núñez-González MA, Rivera-Guillén MA, Serrano-Gallardo LB, Rivas-Morales C. Actividad antibacteriana y citotóxica de Leucophyllum frutescens (Berl) I.M. Johnst del Norte de México contra Staphylococcus aureus de aislados clínicos. Rev Mex Cienc Farm. 2013;44(2):24-30.

24. Almeida, RBA, Akisue G, Cardoso LML, Junqueira JC, Jorge AOC. Antimicrobial activity of the essential oil of Cymbopogon citratus (DC) Stapf. on Staphylococcus spp., Streptococcus mutans and Candida spp. Rev Bras Plantas Med. 2013;15(4):474-482. DOI: 10.1590/S151605722013000400002

25. Tessarollo NG, Carrijo LC, Barbosa MO, Almeida HO, Pereira THA, Magalhães Jr MJ, Leite JPV, Pereira PRG, Baracat-Pereira MC. Fitodefensivos em plantas medicinais: macromoléculas hidrofílicas de folhas de mil folhas (Achillea millefolium L.) inibem o crescimento in vitro de bactérias fitopatogênicas. Rev Bras Plantas Med. 2013;15(2):180-187. DOI: 10.1590/S151605722013000200003 
26. Violante IMP, Hamerski L, Garcez WS, Batista AL, Chang MR, Pott VJ, Garcez FR. Antimicrobial activity of some medicinal plants from the cerrado of the central-western region of Brazil. Braz J Microbiol. 2012;43(4):13021308. DOI: $10.1590 / \mathrm{S} 1517-83822012000400009$

27. Modarresi-Chahardehi A, Ibrahim D, Fariza-Sulaiman S, Mousavi L. Screening antimicrobial activity of various extracts of Urtica dioica. Rev Biol Trop. 2012; 60(4):1567-1576.

28. Millezi AF, Caixeta DS, Rossoni DF, Cardoso MG, Piccoli RH. In vitro antimicrobial properties of plant essential oils Thymus vulgaris, Cymbopogon citratus and laurus nobilis against five important foodborne pathogens. Ciênc Tecnol Aliment. 2012;32(1):167-72. DOI: 10.1590/S0101-20612012005000021

29. Chakravorty S, Rayner MK, Koning CB, Vuuren SF, Otterlo WAL. Synthesis and antimicrobial activity of the essential oil compounds (E)- and (Z)-3-hexenyl nonanoate and two analogues. S Afr J Chem. 2012;65:202-205.

30. Tangarife-Castaño V, Correa-Royero J, Zapata-Londoño B, Durán C, Stanshenko E, Mesa-Arango AC. Anti-Candida albicans activity, cytotoxicity and interaction with antifungal drugs of essential oils and extracts from aromatic and medicinal plants. Infectio. 2001;15(3):160167. DOI: 10.1016/S0123-9392(11)70080-7

31. Santos VL, Souza MFV, Batista LM, Silva BA, Lima MS, Souza AMF, Barbosa FC, Catão RMR. Avaliação da atividade antimicrobiana de Maytenus rigida Mart. (Celastraceae). Rev Bras Plantas Med. 2011;13(1):68-72. DOI: $10.1590 / \mathrm{S} 1516-05722011000100010$

32. Vite-Posadas JA, Brechu-Franco AE, Laguna-Hernández G, Osuna-Fernández HR. Morphoanatomical characterization and antimicrobial activity of Tillandsia imperialis (bromeliaceae). Polibotánica. 2011; s.v.(31): 20-29.

33. Correa-Royero J, Tangarife V, Durán C, Stashenko E, Mesa-Arango A. In vitro antifungal activity and cytotoxic effect of essential oils and extracts of medicinal and aromatic plants against Candida krusei and Aspergillus fumigates. Rev Bras Farmacogn. 2010;20(5):734-741. DOI: S0102-695X2010005000021

34. Höfling JF, Anibal PC, Obando-Pereda GA, Peixoto IAT, Furletti VF, Foglio MA, Gonçalves R. Antimicrobial potential of some plant extracts against Candida species. Braz J Biol. 2010;70(4):1065-1068. DOI: 10.1590/ S1519-69842010000500022

35. Salinas-Sánchez DO, Najera GLA, Rivera IL, Ramírez OD, Cisneros MGV, García VMN. Antimicrobial activity of medicinal plants from the Huautla Sierra Biosphere Reserve in Morelos (Mexico). Polibotánica. 2009; S.v(28):213-225.

36. Mendonça DE, Onofre SB. Atividade antimicrobiana do óleo-resina produzido pela copaiba - Copaifera multijuga Hayne (Leguminosae). Rev bras farmacogn. 2009;19(2b):577-581. DOI: 10.1590/S0102695X2009000400012

37. Alves PM, Queiroz LMG, Pereira JV, Pereira MSV. Atividade antimicrobiana, antiaderente e antifúngica in vitro de plantas medicinais brasileiras sobre microrganismos do biofilme dental e cepas do gênero Candida. Rev Soc Bras Med Trop. 2009;42(2):222-224. DOI: 10.1590/ S0037-86822009000200028

38. Silva Junior IE, Filho VC, Zacchino AS, Lima JCS, Martins DTOS. Antimicrobial screening of some medicinal plants from Mato Grosso Cerrado. Rev Bras Farmacogn. 2009;19(1b):242-248. DOI: 10.1590/S0102695X2009000200011

39. Prestes LS, Frascolla R, Santin R, Santos MAS, Schram RC, Rodrigues MRA, Schuch LFD, Meireles MCA. Actividad de extractos de orégano y tomillo frente a microorganismos asociados con otitis externa. Rev Cubana Plant Med. 2008;13(4):0-0.

40. Valdés AFC, Martínez JM, Lizama RS, Vermeersch M, Cos P, Maes L. In vitro anti-microbial activity of the Cuban medicinal plants Simarouba glauca DC, Melaleuca leucadendron L and Artemisia absinthium L. Mem Inst Oswaldo Cruz. 2008;103(6):615-618. DOI: 10.1590/ S0074-02762008000600019

41. Aguiar JS, Costa MCC D, Nascimento SC, Sena KXFR. Atividade antimicrobiana de Lippia alba (Mill.) N. E. Brown (Verbenaceae). Rev Bras Farmacogn. 2008;18(3):436-440. DOI: 10.1590/S0102$695 \times 2008000300018$

42. Nogueira JCR, Diniz MFM, Lima EO. Atividade antimicrobiana in vitro de produtos vegetais em otite externa aguda. Rev Bras Otorrinolaringol. 2008;74(1):118-24. DOI: $10.1590 / \mathrm{S} 0034-72992008000100019$

43. Ushimaru PI, Silva MTN, Stasi LCD, Barbosa L, Junior AF. Antibacterial activity of medicinal plant extracts. Braz J Microbiol. 2007;38(4):717-719. 10.1590/S151783822007000400024

44. Silva JG, Souza IA, Higino JS, Siqueira-Junior JP, Pereira JV, Pereira MSV. Atividade antimicrobiana do extrato de Anacardium occidentale Linn. em amostras multiresistentes de Staphylococcus aureus. Rev Bras Farmacogn. 2007;17(4):572-577. DOI: 10.1590/S0102$695 \times 2007000400016$

45. Zampini IC, Cudmani N, Isla MI. Actividad antimicrobiana de plantas medicinales argentinas sobre bacterias antibiótico-resistentes. Acta Bioquím Clín. Latinoamer. 2007;41(3):385-393.

46. Scorzoni L, Benaducci T, Almeida AMF, Silva DHS, Bolzani VS, Mendes-Giannini M.S. Comparative study of disk diffusion and microdilution methods for evaluation of antifungal activity of natural compounds against medical yeasts Candida spp and Cryptococcus sp. Rev Ciênc Farm Básica Apl. 2007;28(1):25-34.

47. Klancnik A, Piskernik S, Jersek B, Mozina SS. Evaluation of diffusion and dilution methods to determine the antibacterial activity of plant extracts. J Microbiol Methods. 2010;81(2):121-126. DOI: 10.1016/j.mimet.2010.02.004

48. Alves EG, Vinholis AHC, Casemiro LA, Niege Furtado AJC, Silva MLA, Cunha WR, Martins CHG. Estudo comparativo de técnicas de screening para avaliação da atividade anti-bacteriana de extratos brutos 
de espécies vegetais e de substâncias puras. Quím Nova. 2008;31(5):1224-1229. DOI: 10.1590/S010040422008000500052

49. CLSI, Methods for Dilution Antimicrobial Susceptibility Tests for Bacteria That Grow Aerobically; Approved Standard-Ninth Edition. CLSI document M07-A9. Wayne, PA: Clinical and Laboratory Standards Institute; 2012a.

50. CLSI, Reference Method for Broth Dilution Antifungal Susceptibility Testing of Yeasts; Fourth Informational Supplement. CLSI document M27-S4. Wayne, PA: Clinical and Laboratory Standards Institute; 2012b.

51. Nascimento PFC, Nascimento ACI, Rodrigues CS, Antoniolli ÂR, Santos PO, Júnior AMB, Trindade RC. Atividade antimicrobiana dos óleos essenciais: uma abordagem multifatorial dos métodos. Rev Bras Farmacogn. 2007;17(1):108-113. DOI: $10.1590 / \mathrm{S} 0102-$ 695X2007000100020

52. Ansel HC, Norred WP, Roth IL. Antimicrobial activity of dimethyl sulfoxide against Escherichia coli, Pseudomonas aeruginosa and Bacillus megaterium. J Pharm Sci. 1969; 58(7):836-839. DOI: 10.1002/jps.2600580708
53. Li JL, Lunga PK, Zhao YL, Qin XJ, Yang XW, Liu YP, Luo XD. Antibacterial constituents from Melodinus suaveolens. Chin J Nat Med. 2015;13(4):307-310. DOI: 10.1016/S1875-5364(15)30020-0

54. Chung PY, Navaratnam P, Chung LY. Synergistic antimicrobial activity between pentacyclic triterpenoids and antibiotics against Staphylococcus aureus strains. Ann Clin Microbiol Antimicrob. 2011;10(25):1-6. DOI: 10.1186/1476-0711-10-25

55. Martins A, Vasas A, Viveiros M, Molnár J, Hohmann J, Amaral L. Antibacterial properties of compounds isolated from Carpobrotus edulis. Int $\mathrm{J}$ Antimicrob Agents. 2011;37(5):438-444. DOI: 10.1016/j.ijantimicag.2011.01.016.

56. Rivero-Cruz JF, Sánchez-Nieto S, Benítez G, Casimiro X, Alvarado CI, Molina AR, Rivero-Cruz B. Antibacterial compounds isolated from Byrsonima crassifólia. Rev Latinoamer Quím, 2009;37(2):155-163.

57. Maciel MAM, Pinto AC, Veiga Jr VF, Grynberg NF, Echevarria A. Plantas medicinais: a necessidade de estudos multidisciplinares. Quím Nova. 2002;25(3):429-438. DOI: $10.1590 / \mathrm{S} 0100-40422002000300016$ 